\title{
In silico analysis of high affinity potassium transporter (HKT) isoforms in different plants
}

\author{
Mahbobeh Zamani Babgohari ${ }^{1}$, Esmaeil Ebrahimie ${ }^{2,3^{*}}$ and Ali Niazi
}

\begin{abstract}
Background: High affinity potassium transporters (HKTs) are located in the plasma membrane of the vessels and have significant influence on salt tolerance in some plants. They exclude $\mathrm{Na}^{+}$from the parenchyma cells to reduce $\mathrm{Na}^{+}$concentration. Despite many studies, the underlying regulatory mechanisms and the exact functions of HKTs within different genomic backgrounds are relatively unknown. In this study, various bioinformatics techniques, including promoter analysis, identification of HKT-surrounding genes, and construction of gene networks, were applied to investigate the HKT regulatory mechanism.

Results: Promoter analysis showed that rice HKTs carry ABA response elements. Additionally, jasmonic acid response elements were detected on promoter region of TmHKT1;5. In silico synteny highlighted several unknown and new loci near rice, Arabidopsis thaliana and Physcomitrella patent HKTs, which may play a significant role in salt stress tolerance in concert with HKTs. Gene network prediction unravelled that crosstalk between jasmonate and ethylene reduces AtHKT1;1 expression. Furthermore, antiporter and transferase proteins were found in AtHKT1;1 gene network. Interestingly, regulatory elements on the promoter region of HKT in wild genotype (TmHKT1;5) were more frequent and variable than the ones in cultivated wheat (TaHKT1;5) which provides the possibility of rapid response and better understanding of environmental conditions for wild genotype.

Conclusion: Detecting ABA and jasmonic acid response elements on promoter regions of HKTs provide valuable clues on underlying regulatory mechanisms of HKTs. In silico synteny and pathway discovery indicated several candidates which act in concert with HKTs in stress condition. We highlighted different arrangement of regulatory elements on promoter region of wild wheat $(T M H K T 1 ; 5)$ compared to bread wheat $(T a H K T 1 ; 5)$ in this study.
\end{abstract}

Keywords: HKT, Gene network, Promoter, Regulatory elements, In silico synteny, Pathway discovery

\section{Introduction}

Under salinity stress, the uptake of $\mathrm{Na}^{+}$into cells occurs through multiple $\mathrm{Na}^{+}$-permeable cation channels/transporters, such as outward and inward-rectifying $\mathrm{K}^{+}$-selective channels, in particular non-selective cation channels in the plasma membrane [1]. Loading of xylem vessels with $\mathrm{Na}^{+}$ results in its upward transportation via the transpiration system [2].This transport triggers ion toxicity when the cytoplasmic concentration of $\mathrm{Na}^{+}$reaches to threshold level [2].

Little is known about $\mathrm{Na}^{+}$excluding proteins in plants. $H K T s$ are a large superfamily of transporters. They share

\footnotetext{
* Correspondence: esmaeil.ebrahimie@adelaide.edu.au

${ }^{2}$ Department of Crop Production \& Plant Breeding, College of Agriculture,

Shiraz University, Shiraz, Iran

${ }^{3}$ School of Molecular \& Biomedical Science, The University of Adelaide, Adelaide, Australia

Full list of author information is available at the end of the article
}

sequential and functional similarities with the $\operatorname{TrkH} / \mathrm{KtrB}$ group of cation transporters in bacteria and fungi [3,4]. It has been proposed that these transporters play crucial roles in salinity tolerant via removal of $\mathrm{Na}^{+}$from the xylem during salinity stress [1,2].

In silico promoter analysis can produce valuable information about the function and signalling of a gene. The superiority of an $H K T$ homologue to other homologues can actually be related to the superior promoter structure, rather than the gene structure. Regarding the unknown role of $H K T$ promoters, in silico promoter analysis can provide valuable information. The regulatory elements in promoters, such as transcription factor binding sites, are organized into distinct modules that control expression in many genes. Thus, the identification of regulatory elements is vital for the recognition of gene expression patterns [5]. 
The conserved orientation of HKTs and surrounding genes on a chromosome has not been addressed in previous studies. Identification of comparative genetic maps through in silico synteny can provide the opportunity to acquire information about the evolution and function of a gene cluster via cytogenetic events. It should be noted that the specific orientation of genes in a particular region of a chromosome is commonly associated with particular functions of those genes [6].

In addition to promoter and in silico synteny, network discovery based on available transcriptomics data as well as text mining can be used to understand the function and regulatory mechanisms of HKTs. Construction of gene networks is a powerful tool in detection of genes involved in specific processes, such as biotic and abiotic stress [7]. Recognizing relationships between co-expressed genes and illustrating the involved pathways provide valuable clues on the effect and the role of gene of interest. However, the physiological functions, gene networks, and signalling pathways related to $H K T$ transporters have not yet been completely clarified [8].

In the present study, bioinformatics analysis was employed to illustrate the functional pathways related to $H K T$ transporters in plants and to discover the $H K T$ homologues. The promoter regions of $H K T$ isforms were analyzed. Moreover, in silico synteny was studied as the exact determination of orthology is significant in comparative genomics and biological processes. HKTgene network was built for the first time using available microarray data in order to predict the interacting genes and their possible functions in the stress condition.

\section{Material and methods}

\section{Promoter analysis}

The sequences of all available $H K T s$ in wheat, wild wheat relative (Triticum monococcum), rice and Physcomitrella patens were downloaded from the NCBI (ncbi.nlm.nih.gov) database. The rice sequences were: OsHKT2;1 (AB061311), OsHKT2;2 (AB061313), OsHKT2;3 (AJ491820), OsHKT1;1 (AJ491816), OsHKT1;3 (AJ491818), OsHKT1;4 (AK120889), OsHKT1;5 (EF373553), and OsHKT2;4 (AJ491855). One kb upstream (from the start codon) of the genes were extracted as promoter sequences using Phytozome database (http:// www.phytozome.net/) and Osiris database (http://www.bioinformatics2.wsu.edu/cgi-bin/Osiris/cgi/home.pl). As there was no available database for promoter identification in wheat or wild wheat, a thesis published by Byrt in 2008 was used for TaHKT1;5 and TmHKT1;5 [9]. The putative promoter sequences of $H K T$ genes in rice, bread wheat and T. monococcum were compared with known cisregulatory elements in the collection of the PLANT CARE database (http://bioinformatics.psb.ugent.be/webtools/ plantcare/html/). The cis-regulatory elements were listed and counted for each promoter. Promoter sequences of rice $H K T s$ were also analyzed through the Osiris database where we used rice HKTs accession numbers to find the transcription factor binding sites across the promoter regions. Using this database, significant regulatory elements were selected at the 0.05 probability level (based on Fisher's exact test) to discriminate the transcription factors which have high binding possibility to promoters.

\section{Finding neighbouring genes (synteny analysis)}

Most of the genomic data, stored and publicly available in EMBL and NCBI databases, are without extensive synteny visualization tools [6]. HKT orthologs, initially compiled from BLAST searches of sequences of Arabidopsis thaliana, rice and physcomitrella patent, were extracted from the phytozome database (phytozomewww.phytozome.net). A synteny-based approach was used to identify genes adjacent to $H K T s$. In addition, up to 15 genes up and downstream of rice HKTs were detected using the Gramene database (http://www.gramene.org/genome_browser/index.html).

\section{Gene network discovery for AtHKT1;1}

In this study, 2 sources of microarray data were retrieved for analysis:

\section{Single selected microarray experiment from "Plant Expression Database"}

Micoarray experiment was selected from "Plant Expression Database" (http://www.plexdb.org/). At first, probeset ID of AtHKT1;1 (255812_at) was retrieved from affymetrix database (http://www.affymetrix.com/estore/). Then, different deposited microarray experiments in "Plant Expression Database" were mined using AtHKT1;1 probeset ID. Finally, microarray experiment (Microarray ATH1-121501) considering cross-talk between jasmonate and ethylene signalling in Arabidopsis seedlings was selected. In this experiment, 3 Arabidopsis strains (Col-0, coil-2, and ein3eil1) were treated by Mock and MeJA. The reason for choosing this experiment was that it included two hormones, which could unravel $H K T$ expression pattern, its coexpressed genes, and its genetic interaction network.

Then, the data of this experiment was analyzed using pathway studio 9 and ResNet5.0 database. Pathway Studio is a commercial product for pathway analysis, containing a comprehensive database of protein-protein relationships extracted from literature using MedScan an entirely automated biomedical information extraction engine [10].

\section{Multiple microarray experiments extracted from ATTED-II database for co-expression network analysis}

The co-expression of genes involved in the AtHKT1;1 process was also explored in ATTED-II database (http:// atted.jp/) [11]. Source of GeneChip data in ATTED-II database is TAIR (http://arabidopsis.org/index.jsp). For 
construction of co-expressed network of AtHKT1;1, 58 microarray experiments and 1388 array slides were used. This database collects gene expression data in Arabidopsis from a wide range of microarray experiments. ATTED-II database employs Mutual Ranking (MR score) for coexpresstion analysis instead of Pearson correlation test. Co-expressed of HKT1;1 in abiotic, biotic, hormone and light experiments were extracted $[12,13]$.

\section{Comparative statistics of regulatory elements on promoter regions of TaHKT1;5 (bread wheat) and TmHKT1;5 (wild wheat)}

To have a better understanding of differential arrangement of regulatory elements on the promoter region of $H K T$ in bread wheat verses its wild relatives, the predicted regulatory elements (Table 1) were compared between TaHKT1;5 and TmHKT1;5 in both aspects of central and variation tendencies.

\section{Result and discussion}

Comparative Study of Promoter Regions between HKT1;5-A and $H K T 1 ; 5-D$

Many studies have shown that $H K T$ expression is different among various cell types of plants [2,14]. Moreover, different isoforms of $H K T$ function differently. For instance, it has been reported that TmHKT1;5-A (HKT isoform in Triticum monococcum) decreased $\mathrm{Na}^{+}$concentrations in leaf blades and sheaths to a greater extent than TmHKT1;4-A [2]. In our previous study [15], we measured expression pattern of $H K T 1 ; 5$ in wheat and its wild relatives (A and D genomes) under salt stress using quantitative-real time PCR technique. The results confirmed that HKT1;5 expression is tissue and genotype

Table 1 Elements present in the promoter region of TaHKT1;5 (bread wheat) and TmHKT1;5 (wild wheat) according to the PLANT CARE database

\begin{tabular}{|c|c|c|c|}
\hline cis element & TmHKT1;5 & TaHKT1;5 & Function \\
\hline CAAT-box & 12 & 7 & Common cis-acting element in promoter and enhancer regions \\
\hline Circadian & 2 & 2 & cis-acting regulatory element involved in circadian control \\
\hline CATT-motif & 2 & 2 & Part of a light-responsive element \\
\hline AE-box & 0 & 1 & Part of a module for light response \\
\hline G-box & 2 & 1 & cis-acting regulatory element involved in light responsiveness \\
\hline MRE & 0 & 1 & myb binding site involved in light responsiveness \\
\hline LTR & 2 & 2 & cis-acting element involved in low-temperature responsiveness \\
\hline TC-rich repeats & 1 & 1 & cis-acting element involved in defence and stress responsiveness \\
\hline ARE & 1 & 0 & cis-acting regulatory element essential for the anaerobic induction \\
\hline Box 4 & 1 & 1 & Part of a conserved DNA module involved in light responsiveness \\
\hline Box 1 & 1 & 1 & Light-responsive element \\
\hline ERE & 1 & 1 & Ethylene-responsive element \\
\hline HSE & 1 & 2 & cis-acting element involved in heat stress responsiveness \\
\hline Skn-1_motif & 1 & 1 & cis-acting regulatory element required for endosperm expression \\
\hline TATA-box & 22 & 18 & Core promoter element approximately -30 of the transcription start \\
\hline ACE & 1 & 1 & cis-acting element involved in light responsiveness \\
\hline I-box & 2 & 0 & Part of a light-responsive element \\
\hline MNF1 & 1 & 1 & Light-responsive element \\
\hline O2-site & 1 & 0 & cis-acting regulatory element involved in zein metabolism regulation \\
\hline ATCT-motif & 1 & 1 & Part of a conserved DNA module involved in light responsiveness \\
\hline CCAAT-box & 1 & 1 & mybHv1 binding site \\
\hline CGTCA-motif & 2 & 1 & cis-acting regulatory element involved in the MeJA-responsiveness \\
\hline Sp1 & 2 & 3 & Light-responsive element \\
\hline TGA-element & 1 & 1 & Auxin-responsive element \\
\hline TGACG-motif & 2 & 1 & cis-acting regulatory element involved in the MeJA-responsiveness \\
\hline GARE-motif & 1 & 1 & Gibberellin-responsive element \\
\hline CCGTCC-box & 0 & 1 & cis-acting regulatory element related to meristem specific activation \\
\hline A-box & 0 & 1 & cis-acting regulatory element \\
\hline
\end{tabular}


dependent [15]. In the mentioned study, earlier expression of $H K T 1 ; 5$ in response to salt stress was observed in leaves of bread wheat rather than roots. Additionally, under high $\mathrm{NaCl}$ level $(200 \mathrm{mM})$ treatment, the allele of D genome $(H K T 1 ; 5-D)$ showed higher expression than the allele of A genome (HKT1;5-A). We found that promoter region of $H K T 1 ; 5-D$ has jasmonic acid response element [15] which this element is not present at $H K T 1 ; 5-A$. In other words, the observed differential expression pattern of $H K T s$ can be explained by differential architecture of promoter regions in the view of existence/absence of regulatory elements. This is an encouraging and mostly unknown topic for future studies.

The results of the PLANT CARE analysis of promoter regions are shown in Table 1 . Interestingly, TATA box and CAAT elements were more frequent in the TmHKT1;5 promoter than TaHKT1;5. The presence and the number of TATA boxes can increase the quantity of gene expression. However, the exact activation of the putative TATA boxes in HKTs has not been investigated in laboratory level.

Some studies suggested that TATA box has a variable position in -100 and -40 nucleotides from the start codon [16-20]. In contrast, other studies argued that the effective region of TATA box is $32 \pm 7$ and can alter up to 50 nucleotides upstream of the transcription initiation site [16-19]. It seems that position of putative TATA box is variable between different gene promoters.

Interestingly, Sharma and co-workers in 2011 illustrated that the presence of two TATA boxes at positions -59 and -359 and two CCAAT elements sitting at positions of -355 and -590 are involved in strong expression of AlX in Aspergillus Niger [21].

Accordingly, it is possible that each of the predicted TATA boxes in the present study to have positive impact on $H K T$ expression regardless of their positions on the promoter region. Consequently, we supposed that the increased number of TATA boxes in promoter of TmHKT1;5 (wild wheat) compared to TaHKT1;5 (bread wheat) can result in higher expression of TmHKT1;5.

PLANTCARE analysis detected high frequency of TATA boxes around position $-50(-42 /-44 /-50 /-52)$ in TmHKT1;5 while in TaHKT1;5 promoter, the peak of TATA boxes happened around position -30 (-19/ -20/ -22/ -35). In our 2012study, we suggested that in high salinity condition, D genome of TaHKT1;5 is more effective than A genome of TbHKT1;5 and TmHKT1;5 [12]. With respect to the position of TATA boxes in TaHKT1;5 promoter (around position -30), it can be concluded that position of -30 is more important than position of -50 in initiation of transcription under high salinity ( $200 \mathrm{mM}$ condition), conferring higher level of expression in TaHKT1;5 compared to TbHKT1;5. In other words, it seems that at least under high salinity stress, the number of TATA boxes in a specific position of promoter $(-30)$ is more effective than the whole numbers of TATA boxes which are spread across the entire promoter region. It should be noted that TATA boxes in other regions of promoter such as position of -50 in TmHKT1;5 can function in different situations such as other abiotic/biotic stresses. The link between position of TATA box and tissue specific expression is also probable as $T m H K T 1 ; 5$ has significantly higher expression in roots rather than the leaves [22].

Another interesting regulatory element in comparison of TmHKT1;5 with TaHKT1 is CAAT-box (Table 1). CAATbox sequences have proved positive impact on frequency of transcriptional initiation [23]. Interestingly, in silico based analysis of promoter regions in this study revealed the existence of two CAAT-boxes in TmHKT1;5 promoter at the range of -50 to -100 position ( -53 and -76 , respectively). In contrast, CAAT-boxes in promoter of TaHKT1;5 are located out of -100 position from the start codon. Arrangement of CAAT-boxes close to transcription start codon in TmHKT1;5 can help wild wheat in better response to environmental signals trough rapid activation of HKT1.

Since many defensive genes respond to jasmonate, jasmonates are universal signals of defense-related gene expression [24]. In the present study, the jasmonate response element (MeJA) was two times more common in TmHKT1;5 than TaHKT1;5 (Table 1). Jasmonate is involved in plant adaptations to biotic and abiotic stresses, and is accumulated transiently in response to osmotic/salt stress [25].

It has been stated that expression of TbHKT1;5 is higher than TaHKT1;5 under low/medium salt stress (50 and $100 \mathrm{mM} \mathrm{NaCl}$ ) [15]. So, this salinity tolerance in the TbHKT1;5 can be related to some elements in the promoter region such as jasmonate response element (MeJA) that was more frequent in this plant. Interestingly, it has been shown that application of MeJA improves the tolerance under moderate saline stress $(40 \mathrm{mM} \mathrm{NaCl})$ in broccoli, but not in high salinity conditions $(200 \mathrm{mM} \mathrm{NaCl})$ [26], which is in parallel to our assumption about the role of MeJA in TmHKT1;5/ TbHKT1;5 promoter. It is possible that the MeJA and TATA boxes are not active in higher salt conditions, so the expression of $H K T$ decreases in T.boeoticum in high salinity condition. However, the important point is that jasmonate response element can cause salinity tolerance, a positive trait in salt environments at least in low/moderate salinity conditions.

The promoter analysis also showed that HSE, a cisacting element involved in heat stress responsiveness, is more frequent in TaHKT1;5-D than TmHKT1;5. Thus, it is likely that these $H K T \mathrm{~s}$ are expressed in response to high temperature too.

\section{Promoter analysis of all HKT isoforms in rice}

The promoter analysis was performed in all available rice $H K T$ transporter isoforms, separated into two subfamilies. 
There are two subfamilies of $H K T$ s based on glycine or serine substitution of a residue predicted in the first pore loop of the protein $[27,4]$. The results are divided into two tables based on subfamilies I and II (Table 2 and Table 3). In subfamily I, HKT transporters have a serine residue that determines $\mathrm{Na}^{+}$transport, but subfamily II contains a glycine residue, responsible for $\mathrm{K}^{+}$permeability primarily $[9,14,28]$.

This analysis showed that the HKTs belonging to subfamily I have more of the following motifs than subfamily II: ABA (abscisic acid), auxin, defense responsive, HSE (heat shock element), low temperature response and MYB biding site. However, subfamily II of $H K T$ transporters contains the following motifs more frequently than subfamily I: ethylene, gibberellin and salicylic acid response. The results indicated that there are nine ABRE motifs (ABA response element) involved in the response to $\mathrm{ABA}$ within subfamily I of HKT promoters, while no ABA response motif is present in subfamily II. ABA is a very important hormone involved in signalling of various stresses such as salinity and drought [29]. The presence of ABRE and MYB motifs were also revealed in MtATP6 promoter as an abscisic acid-mediated signalling [30]. At salt conditions, It has been also shown that lack of AtHKT1;1 (HKT isoform in Arabidopsis thaliana) activation in mutated Arabidopsis thaliana leads to $\mathrm{Na}^{+}$accumulation in shoot and its decrease in root, in comparison to control plants [8]. Thus, AtHKT1;1 has a vital role in $\mathrm{Na}^{+}$exclusion from the shoot to root [31]. OsHKT1;5 (HKT1;5 isoform in rice) is an ortholouge of AtHKT1;1 associated with $\mathrm{Na}^{+}$exclusion from the xylem and has the same role in rice [32]. Nevertheless, In the other study, Kader and co-workers in 2006 showed that the expression of OsHKT1;1, another $H K T$ isoform in rice, was unexpectedly high in salt sensitive rice cv. BRRI Dhan29 than that in the salt tolerant $\mathrm{cv}$. Pokkali. This result also indicated that in salt sensitive cultivar, OsHKT1;1 expression happened in earlier sampling time than the salt tolerant one. Therefore, OsHKT1;1 is vital in $\mathrm{Na}^{+}$influx into plant cell compared to OsHKT1;5 and AtHKT1;1 [33]. Altogether, different $H K T$ isoforms have variety of expressions and actions at different tissues for unknown reasons [15]. We assume that presence of ABA element in the promoter of these genes can be a reason for salinity tolerance. Interestingly, the core promoter (TATA box), at the position of -50 and -30 nucleotides upstream from start codon, was more frequent in subfamily I than subfamily II. As the function of TATA box is in transcription initiation, increased expression of subfamily I HKT transporters is expected compared to subfamily II. However, according to different studies, it seems that sometimes subfamily II of $H K T$ transporters even in salt condition have more expresstion [8]. As a result, it appears that subfamily I primarily may be more critical in many stress responses in plants as its activation is related to $\mathrm{Na}^{+}$ transport. On the other hand, it is possible that by continuing stress, the expression of subfamily II will increase, leading to $\mathrm{K}^{+}$transport into the plant cells, and reducing salinity damages $[33,34]$.

OsHKT2;1, belonging to subfamily II, exhibited higher expression in salt tolerant cv, Pokkali than salt sensitive cv. BRRI Dhan29, particularly in shoot. Consistent with this result, it is clear that OsHKT2;1 involves in $\mathrm{K}^{+}$uptake [33]. In this study, Osiris result at $P=0.05$ indicated that all of the significant motifs identified in both subfamilies have ABA response elements. These motifs were observed in $K T 1 ; 1, H K T 1 ; 3$, and HKT1;4, which belong to subfamily I HKTs, as well as $H K T 2 ; 1$, and $H K T 2 ; 2$, which are related to subfamily II (Table 4).

Osiris found the element named Hox1(homeodomain transcription factor) which is a putative member of the leucine-zipper (HD-ZIP) transcription factor class [35] and HD-ZIP which is an ABA-independent motif exist in both subfamilies I and II. Besides the role of ABA in controlling plant responses to salinity, regulating plant responses to drought stress are managed by ABA [36,37]. We suggest a model for motif activation in two pathways related to ABA in Figure 1 in response to drought and salinity stresses since ABA has a central role in both stresses. The next significant element was ABADESI1, which was identified in both $H K T$ subfamilies. ABADESI1 is a motif involved in osmotic responses related to physiological processes and is identified with the RAB16-A gene promoter between the -294 and -52 region of this gene; it confers ABA-dependent expression on the chloramphenicol acetyl-transferase reporter gene in rice [38]. There was also a GC-rich repeat IV motif in the HKT6 promoter. Previous studies have indicated that the GC motif in the RAB 21 gene promoter contains four different GC types at the range of -1 to -200 that are active in response to salinity, drought, and ABA in rice [38]. ABADESI2 is an ABA response element, located between -180 and -160 of the wheat histone $\mathrm{H} 3$ promoter that plays an essential role in drought and salinity stresses [39]. Interestingly, $H K T$ subfamily II had both ABA-dependent and independent elements on the promoter region.

$H K T 1 ; 5$, belonging to subfamily I, significantly upregulates in salinity condition, especially in Aegilops crassa [15]. ABA response elements in subfamily I are more frequent than subfamily II. Consequently, it seems reasonable that this subfamily, even in high salt conditions, is responsible for $\mathrm{Na}^{+}$exclusion rather than $\mathrm{K}^{+}$transport. It appears that the various roles of these subfamilies in $\mathrm{K}^{+}$ and $\mathrm{Na}^{+}$transport and their different functions are related to their special architecture of their promoter regions.

Synteny analysis and identification of novel interacting genes In silico synteny was assessed at rice, Arabidopsis thaliana and Physcomitrella patens HKT loci (Figure 2). The results 
Table 2 Elements present in the promoter regions of subfamily I of HKTs in rice according to the PLANT CARE database

\begin{tabular}{|c|c|c|c|c|c|c|}
\hline Function & Motif & $\begin{array}{l}\text { HKT1;1 } \\
\text { AJ491816 }\end{array}$ & $\begin{array}{l}H K T 1 ; 3 \\
\text { AJ491818 }\end{array}$ & $\begin{array}{l}\text { HKT1;4 } \\
\text { AK10985 }\end{array}$ & $\begin{array}{l}\text { HKT71;4 } \\
\text { AK120889 }\end{array}$ & $\begin{array}{l}\text { HKT81;5 } \\
\text { AK108663 }\end{array}$ \\
\hline \multirow[t]{3}{*}{ ABA } & ABRE & 1 & - & 2 & 2 & - \\
\hline & $\| B$ & - & - & 1 & 1 & - \\
\hline & CE3 & - & - & 1 & 1 & - \\
\hline Salicylic acid & TCA & 2 & 1 & - & - & - \\
\hline Ethylene & ERE & - & - & - & - & - \\
\hline \multirow[t]{2}{*}{ Auxin } & AuxRR-core & 2 & - & - & - & - \\
\hline & TGA & 1 & - & - & - & - \\
\hline \multirow[t]{2}{*}{ Gibberellin } & P-Box & - & - & - & - & - \\
\hline & GARE & - & - & - & - & 1 \\
\hline MYB & MBS & 2 & 2 & 1 & 1 & 1 \\
\hline Defence\&stress & TC-reach & - & 1 & 2 & 2 & 1 \\
\hline Heat stress & HSE & 3 & 3 & - & - & - \\
\hline Low temperature & LTR & 2 & - & - & - & - \\
\hline Fungal elicitor & BOX-W1 & - & 1 & - & - & - \\
\hline Wound & WUN & - & - & 1 & 1 & - \\
\hline Anaerobic & ARE & 1 & 1 & 1 & 1 & - \\
\hline \multirow[t]{2}{*}{ MejA } & TGACG & 2 & - & - & - & 1 \\
\hline & CGTCA & 2 & - & - & - & 1 \\
\hline High transcription level & 5UTR Py-rich stretch & 1 & 1 & - & - & - \\
\hline Meristem expression & CAT-BOX & 2 & - & - & - & - \\
\hline Endosperm expression & SKn-1 & - & 2 & - & 2 & - \\
\hline MYBHv1 & CCAAT-box & - & - & 1 & 1 & - \\
\hline Cell cycle regulation & MSA & - & - & 1 & 1 & - \\
\hline Seed specific regulation & RY-element & - & - & 2 & 2 & - \\
\hline Endosperm negative expression & AACA & - & - & - & - & 1 \\
\hline Zein metabolism regulation & O2-site & - & 1 & 1 & 1 & 2 \\
\hline Anoxic specific inducibility & GC & - & - & - & - & - \\
\hline Circadian control & Circadian & - & - & 1 & 1 & 2 \\
\hline Differentiation of the palisade & HD-Zip1 & 2 & - & - & - & - \\
\hline Leaf morphology development & HD-Zip2 & 1 & - & - & - & - \\
\hline \multirow[t]{12}{*}{ Protein binding site } & HD-Zip3 & - & - & - & - & 1 \\
\hline & BOXIII & 1 & 1 & - & - & - \\
\hline & ATCT & - & - & - & - & - \\
\hline & I-box & - & 1 & 2 & - & - \\
\hline & $\mathrm{TCT}$ & - & - & - & - & - \\
\hline & G-BOX & - & - & 2 & 2 & - \\
\hline & GAG & 1 & - & 2 & 2 & - \\
\hline & GT1 & - & - & - & - & 1 \\
\hline & ATC & 1 & - & - & - & - \\
\hline & 1 & - & - & - & - & 1 \\
\hline & CATT & - & 1 & - & - & - \\
\hline & F-box & - & - & - & - & - \\
\hline
\end{tabular}



database (Continued)

\begin{tabular}{|c|c|c|c|c|c|c|}
\hline \multirow[t]{12}{*}{ Light responsive } & Chs-CMA2a & - & - & - & - & - \\
\hline & GATA & - & 1 & - & - & - \\
\hline & ACE & - & - & 1 & 1 & - \\
\hline & $\mathrm{BOX} 4$ & - & - & 1 & 1 & 2 \\
\hline & GA & - & - & - & 1 & - \\
\hline & LAMP & - & - & 2 & 1 & - \\
\hline & MRE & - & - & 1 & 1 & - \\
\hline & SP1 & - & - & 1 & 1 & 1 \\
\hline & MNF1 & - & - & - & - & 2 \\
\hline & TGGCA & - & - & - & - & 1 \\
\hline & As-2-box & - & - & - & - & - \\
\hline & AE-BOX & - & - & 1 & 1 & - \\
\hline Promoter \& enhancer & CAAT-box & 36 & 28 & 18 & 18 & 18 \\
\hline Core promoter & TATA & 10 & 27 & 51 & 51 & 56 \\
\hline
\end{tabular}

indicated that three loci (Os04g51809.2, Os06g48780.1, and Os06g48790.1) exist upstream of the HKT isoforms in rice, and there are six loci downstream of the mentioned genes (Os02g07830.1, Os02g07840.1, Os02g07840.1, Os02g07840.2, Os02g07850.1, Os01g34860.1, and Os01g34870.1). It is probable that $H K T$ gene neighbours may have a similar function as HKTs. The other loci identified based on in silico synteny are currently unidentified. As a result, we proposed that these loci may play a role in the response to salt or drought stress, similar to $H K T s$ or, likely, other unknown $H K T$ isoforms that will be found in the future. It should be noted that genes with similar function usually cluster together along the chromosomes during the evolution. The point is that HKTs belonging to subfamily I are not together with subfamily II. Thus, having different and specific cis elements is acceptable in HKTs promoter region. Remarkably, analysis of those loci indicated that two loci, Os02g07840.2 and Os01g34860.1, near OsHKT1;3 and OsHKT2;3, respectively, are b-ZIP protein and leucine zipper protein-like. Thus, the result of synteny is in agreement with promoter analysis results showing that $\mathrm{ABA}$ response elements are the most important motifs in $H K T$ promoters. Therefore, it is possible that ABA can also influence loci close to HKTs. Interestingly, HKT2;1 and $H K T 2 ; 4$ are located on chromosome 6, and there are three homologs of HKT1;1 and one of HKT1;4 neighbouring each other on chromosome 4 (Figure 2). Interestingly, HKTs belonging to each subfamily (I or II) are located near to each other on chromosomes.

There is one locus upstream and one locus downstream of the HKT gene in Arabidopsis thaliana, which are AT4G10300.1 and AT4G10320.1, respectively. Arabidopsis thaliana has one HKT isoform (AtHKT1;1) which is responsible for excluding $\mathrm{Na}^{+}$from phloem to xylem cells
[8]. This isoform plays a very important role in salinity tolerance in this plant. Physcomitrella patent is a salttolerant plant. There are two loci (Pp1s63_162v6.1 and Pp1s63_163v6.1) upstream and one locus (Pp1s63_165v6.1) downstream of the $H K T$ gene in this plant (Figure 2). We suggest that these loci probably have an essential duty to improve tolerance under some stresses in Arabidopsis thaliana and Physcomitrella patent.

The results of Gramene database showed that many genes close to rice HKTs are unidentified. The known ones, located $15 \mathrm{~kb}$ up- and downstream of $H K T s$, are shown in Table 5 which encode transporters, kinases and proteases. It has been suggested that protein kinases are involved in signal transduction and are activators of SOS (salt overlay sensitive) genes [15]. Regarding the remarkable role of kinase proteins in SOS processes under osmotic response [40-42], it is possible that these proteins influence $H K T s$ as well.

\section{Pathway Discovery in AtHKT1;1 and Identification of Co-expressed Genes \\ Network of AtHKT1;1 in response to jasmonate and ethylene hormones}

Based on the selected microarray experiment from "Plant Expression Database" (Microarray ATH1-121501) which evaluates cross-talk between jasmonate and ethylene on Arabidopsis seedlings, network of interacting proteins with AtHKT1;1 was recognized. The main pathway was so complex making it difficult to identify the nearest proteins connected to AtHKT1;1; to detect it easier, the specific pathway associated with proteins in connection with AtHKT1;1 was predicted separately using pathway studio, as shown in (Figure 3A). In this pathway, AtHKT1;1 was down regulated, showing reduction in expression. It 
Table 3 Elements present in the promoter region of all isoforms of subfamily II HKTs in rice according to the PLANT CARE database

\begin{tabular}{|c|c|c|c|c|c|c|}
\hline \multirow[t]{2}{*}{ Function } & \multirow[t]{2}{*}{ Motif } & \multirow{2}{*}{$\begin{array}{l}\text { HKT2;1 } \\
\text { АB061311 }\end{array}$} & \multirow{2}{*}{$\begin{array}{l}\text { HKT2; } 1 \\
\text { AK105132 }\end{array}$} & \multirow{2}{*}{$\begin{array}{l}\text { HKT2;2 } \\
\text { AB061313 }\end{array}$} & \multirow{2}{*}{$\begin{array}{l}\text { HKT2;3 } \\
\text { AJ491820 }\end{array}$} & \multirow{2}{*}{$\begin{array}{l}\text { HKT2;4 } \\
\text { AJ491855 }\end{array}$} \\
\hline & & & & & & \\
\hline \multirow[t]{3}{*}{ ABA } & ABRE & - & - & - & - & - \\
\hline & $\| \mathrm{B}$ & - & - & - & - & - \\
\hline & CE3 & - & - & - & - & - \\
\hline Salicylic acid & TCA & 1 & - & 3 & - & - \\
\hline Ethylene & ERE & - & 1 & - & - & - \\
\hline \multirow[t]{2}{*}{ Auxin } & AuxRR-core & - & - & - & - & - \\
\hline & TGA & - & - & - & - & - \\
\hline \multirow[t]{2}{*}{ Gibberellin } & P-Box & 1 & - & - & - & - \\
\hline & GARE & - & - & - & - & 1 \\
\hline MYB & MBS & 1 & 3 & - & 1 & 2 \\
\hline Defence\&stress & TC-reach & - & 2 & 2 & - & - \\
\hline Heat stress & HSE & - & - & - & - & - \\
\hline Low temperature & LTR & - & - & - & 1 & - \\
\hline Fungal elicitor & BOX-W1 & - & - & - & - & - \\
\hline Wound & WUN & - & - & 1 & 1 & - \\
\hline Anaerobic & ARE & 1 & - & 1 & 1 & - \\
\hline \multirow[t]{2}{*}{ MejA } & TGACG & 2 & 1 & - & - & 1 \\
\hline & CGTCA & - & 1 & - & - & 1 \\
\hline High transcription level & 5UTR Py-rich stretch & - & - & 1 & - & - \\
\hline Meristem expression & CAT-BOX & - & - & 1 & 1 & - \\
\hline Endosperm expression & SKn-1 & 3 & 2 & 5 & 3 & 4 \\
\hline MYBHv1 & CCAAT-box & - & 1 & - & - & - \\
\hline Cell cycle regulation & MSA & - & - & - & - & - \\
\hline Seed specific regulation & RY-element & - & - & - & - & - \\
\hline Endosperm negative expression & AACA & - & - & - & - & - \\
\hline Zein metabolism regulation & O2-site & - & - & 1 & 2 & 2 \\
\hline Anoxic specific inducibility & GC & - & 1 & - & - & - \\
\hline circadian control & Circadian & - & 1 & - & - & - \\
\hline Differentiation of the palisade & HD-Zip1 & 1 & - & 2 & - & - \\
\hline Leaf morphology development & HD-Zip2 & 1 & - & 1 & - & - \\
\hline \multirow[t]{10}{*}{ Protein binding site } & HD-Zip3 & 1 & - & - & - & - \\
\hline & BOXIII & - & - & - & 1 & - \\
\hline & ATCT & 1 & - & 1 & - & - \\
\hline & I-box & 1 & 1 & 2 & 1 & 1 \\
\hline & TCT & 1 & - & 1 & - & - \\
\hline & G-BOX & - & 1 & 2 & - & - \\
\hline & GAG & - & - & 1 & 2 & - \\
\hline & GT1 & - & - & 1 & 2 & - \\
\hline & ATC & - & - & - & - & - \\
\hline & TGG & - & - & - & - & - \\
\hline \multirow[t]{2}{*}{ Light responsive } & CATT & - & - & - & 3 & 1 \\
\hline & F-box & - & 1 & - & - & - \\
\hline
\end{tabular}




\begin{tabular}{|c|c|c|c|c|c|c|}
\hline & Chs-CMA2a & - & 1 & - & - & - \\
\hline & GATA & - & - & - & - & 2 \\
\hline & ACE & - & - & - & - & - \\
\hline & BOX4 & - & - & - & - & 1 \\
\hline & GA & - & - & - & - & - \\
\hline & LAMP & - & - & - & - & - \\
\hline & MRE & - & - & - & - & - \\
\hline & SP1 & 2 & - & - & - & - \\
\hline & MNF1 & - & - & - & - & - \\
\hline & TGGCA & - & - & - & - & - \\
\hline & As-2-box & - & - & - & - & 1 \\
\hline & AE-BOX & 1 & 1 & 1 & - & - \\
\hline Promoter \& enhancer & CAAT-box & 21 & 19 & 39 & 22 & 24 \\
\hline Core promoter & TATA & 14 & 1 & 34 & 22 & 54 \\
\hline
\end{tabular}

appears that using jasmonate and ethylene decreased the function of AtHKT1;1 in Arabidopsis seedlings. The question is, why was AtHKT1;1 down regulated? As we discussed before, the most important hormone in $H K T$ activation process is ABA. This idea was also confirmed through promoter analysis of $H K T$ genes. Therefore, it is reasonable that other hormones are not very active rather than ABA where HKTs are expressed strongly. On the other hand, combined effect of two hormones can be another reason of decreasing AtHKT1;1 expression [43]. As a very interesting point, the MeJA response element was found in the promoter region of TmHKT1;5 as well, indicating that this transcription factor is not convenient in high salinity milieus, and proving the result of network discovery in which the AtHKT1;1 was down regulated under treatment of MeJA.

However, the results indicated that some of the proteins in the AtHKT1;1 pathway were involved in ion transporting (Table 6). This result was predictable due to the role of HKTs as transporters. Although AtHKT1;1 was down-regulated in the selected experiment, it seems that other transporter genes were up-regulated instead. Perhaps in this situation (two hormones crosstalk) there was not any need to express AtHKT1;1.

Table 4 Significant elements in the promoters of all HKT isoforms of rice at $\boldsymbol{P}=\mathbf{0 . 0 5}$ according to the Osiris database

\begin{tabular}{cccc}
\hline Cis-element & Locus & Gene & Function \\
\hline ABADESI1, hox1 & Os06g0701700 & HKT1;1, HKT2;1, HKT2;2 & ABA response \\
ABADESI2 & Os04g0607600 & HKT1;4 & ABA response \\
GCrichrepeatIV & Os02g0175000 & HKT1;3 & ABA response \\
\hline
\end{tabular}

\section{Co-expressed network of AtHKT1;1}

In addition to prediction the down regulated network of proteins together with AtHKT1;1, the genes coexpressed with AtHKT1;1 were compared to find out what genes are most likely active when $A t H K T 1 ; 1$ is expressed. In this case, despite the other pathway, the up regulated genes with induced expression are shown. It was found that the three nearest proteins in the above gene pathway were TPS14, CYP71B31 and F15E12.22, which are a terpene synthase, a protein involved in oxidation reduction and an acyl-activating enzyme. However, presence of oxidation reductase protein shows that the possibility of plant toxicity in some stress conditions is obvious, and some proteins like reductase must decline this danger. Additionally, co-expressing oxidation reductase proteins with $A t H K T 1 ; 1$ attests that the expression of AtHKT1;1 increases $\mathrm{Na}^{+}$in some cell types, and turns the detoxification system on. Other pathway proteins are shown in (Figure 3 and Table 7).

The designed co-expressed network in this study, based on a large amount of publicly available transcriptomics data, significantly elaborated our understanding of the gene interaction and function of AtHKT1;1. Developed databases of gene co-expression analysis which utilize large amount of publicly available transcriptomics data are offering valuable information on relative expression levels of thousands of genes simultaneously which can be exploited for drawing co-expression networks [44]. In fact, large collections of expression data derived from EST, microarray, or RNA-seq platforms contain information about concerted changes in transcript levels these datasets beyond the original purpose of each experiment.

Traditionally, Pearson's correlation coefficient is index of co-expression where " 1 " indicates strong Relation and 


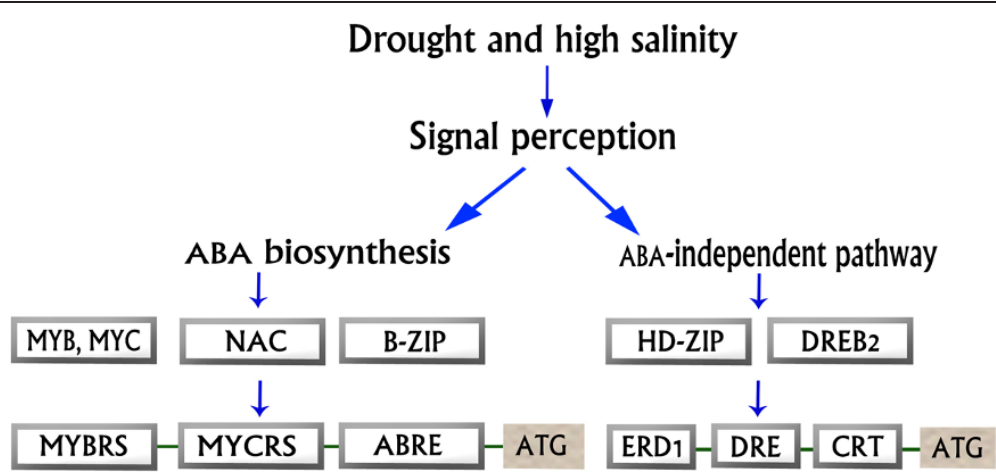

Figure 1 Different active transcription factors responding to salinity and drought stresses in ABA-dependent and ABA-independent pathways during the induction of stress genes. Various transcription factors are triggered in response to stresses and mediate their influence on stress genes mainly through two pathways. The transcription factors involved in ABA-dependent pathways are b-ZIP (basic leucin zipper), MYC (myelocytomatosis oncogene) and MYB (myeloblastosis oncogene), NAC (NAM, ATAF1,2 and CUC2), which interact with ABRE (ABA-responsive element) or MYCRS/MYBRS elements in the promoters of stress genes. Members of the ABA-independent pathways include a number of transcription factors, such as HD-ZIP (Homeodomain Leucine Zipper Protein) and DREB2 (DRE-binding protein), which are activated during salt and drought stresses. ATG means start codon, DRE / CRT (dehydration responsive element/C-repeat) and ERD1 (early responsive to dehydration1.

"0" indicates no relation. In this study, we employed $\mathrm{Mu}-$ tual Ranking (MR) which is a more robust co-expression measurement [45]. MR provides a reliable statistical tool for measurement of co-expression between genes in different transcriptomics experiments.
Regulatory elements on promoter region of TmHKT1;5 (wild wheat) are more frequent and variable than TaHKT1;5 (bread wheat)

Statistics of regulatory elements on the promoter region of TmHKT1;5 and TaHKT1;5 is presented at Table 8.

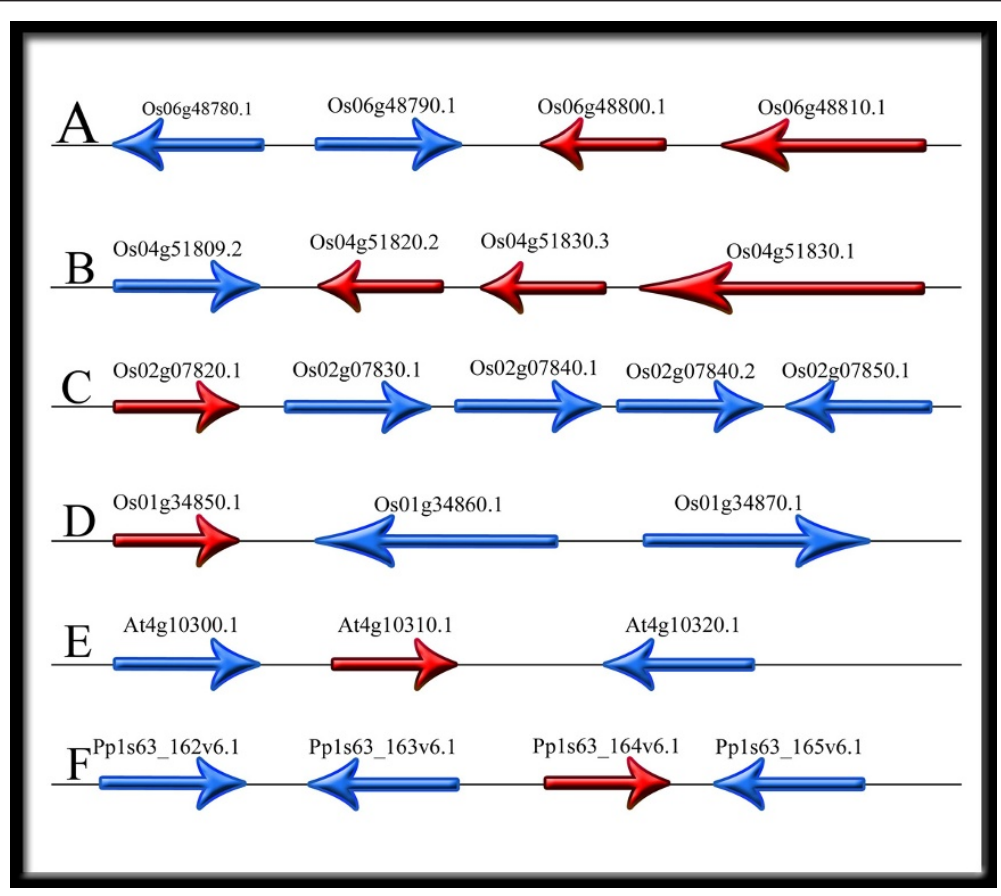

Figure 2 Gene arrangement revealed by in silico synteny analysis of different HKT homologues in rice (A to D), Arabidopsis thaliana (E) and Physcomotrell patens (F) along the chromosome. The red colour indicates HKT homologues along a chromosome, and there are several loci (blue colour), which are largely unidentified, near HKTs. The red loci on chromosome 6 of rice are Os06g48800.1 (HKT2;4) and Os06g48810.1 (HKT2;1). On chromosome 4, Os04g51820.2 and OS04g51830.3 are HKT1;1, and OS04g51830.1 is HKT1;4. On chromosome 2, Os02g 07820.1 is HKT1;3, and on chromosome 1, Os01g34850.1 is HKT2;3. In Arabidopsis thaliana, AT4g10310.1 is HKT1;1, and in Physcomotrell patens, Pp1s63_164v6.1 is HKT1. 
Table 5 In silico synteny indicated known upstream and downstream loci of HKT isoforms in rice using the Gramene database

\begin{tabular}{ccccc}
\hline Locus & down stream & Locus & Upstream & GENE \\
\hline Os01g20160 & skc1 & Os01g20120 & near skc1 & OsHKT1;5 \\
Os02g07830 & Na transporter & Os02g07760 & SSADH & AK062651.1 \\
& & Os02g07780 & CTR1 & HKT1; \\
Os04g51830 & Os02g07790 & & OsHKT1;4 \\
Os04g51950 & Kinase & Os04g51820 & & putative NB-ARC domain- \\
Os06g48650 & (TAP)- kinase & Os06g48520 & (TAP)- kinase & OsHKT2;1 \\
& Protease & Os06g48590 & Serine Blactamse family & OsHKT2;4 \\
\hline
\end{tabular}

A
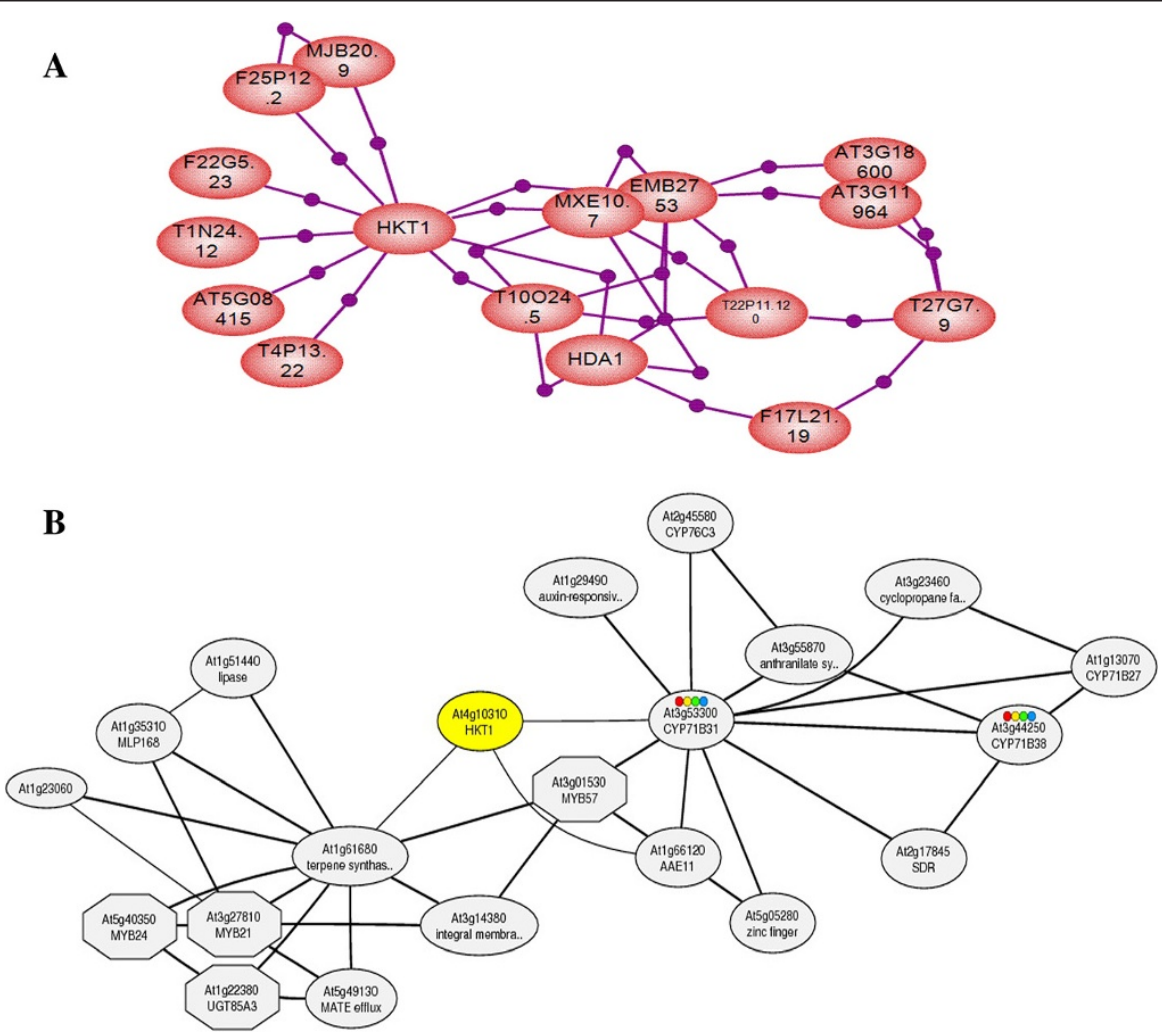

Figure 3 Pathway of AtHKT1;1 and related proteins constructed using pathway studio package. (A) Down regulated genes in the AtHKT1;1 protein pathway are shown. These proteins are related to the AtHKT1;1 protein and are down regulated in the selected microarray experiment, [Microarray ATH1-121501: analyzing crosstalk between jasmonate and ethylene in Col-0, coi1-2, and ein3eil1 strains in two treatments of Mock and MeJA, downloaded from "Plant Expression Database" (http://www.plexdb.org)]. (B) Condition-specific co-expression of genes in the AtHKT1;1 pathway according to the ATTED-II database (http://atted.jp). In ATTED co-expression mining, correlation rank is used rather than Pearson correlation value. Source of GeneChip data in ATTED-II database is TAIR http://arabidopsis.org/index.jsp, and 58 experiments and 1388 array slides are used for drawing this co-expressed network. Octagon shapes mean that the genes they cover are transcription factors while ovals cover genes with different functions. Four colourful circles within oval's of CYP71B31 and CYP71B38 genes are related to various KEGG http:/www.genome.jp/kegg/ pathways which these two genes can be active there, so that red shows Naphthalene and anthracene degradation, yellow shows gammaHexachlorocyclohexane degradation, green shows Limonene and pinene degradation and blue shows Phenylpropanoid biosynthesis pathways. While the figure A shows down regulated protein associated with AtHKT1;1, the up regulated proteins connected to AtHKT1;1 are shown in figure B. 
Table 6 Results of the pathway discovery analysis indicating proteins activated in the AtHKT1;1 network based on microarray analysis of cross-talk between jasmonate and ethylene signalling in Arabidopsis seedlings using pathway studio 9

\begin{tabular}{|c|c|}
\hline Protein & Function \\
\hline Hda1 & Encodes a histone deacetylase involved in jasmonic acid and ethylene-dependent pathogen resistance \\
\hline MJB20.9 & Protein kinase super protein amino acid phosphorylation \\
\hline ATCHX23 & Member of a putative $\mathrm{Na}+/ \mathrm{H}+$ antiporter family \\
\hline F22G5.23 & Arabidopsis thaliana sterol 4-alpha-methyl-oxidase mRNA \\
\hline EMB2753 & Embryo development ending in seed dormancy \\
\hline T1N24.12 & Protein amino acid phosphorylation \\
\hline T10024.5 & Encodes SIN3-like 6, a homologue of the transcriptional repressor \\
\hline KCO4 & Encodes AtTPK4, a member of the Arabidopsis thaliana $K+$ channel family of AtTPK/KCO proteins \\
\hline AT5G08415 & Radical SAM superfamily protein; FUNCTIONS IN: 4 iron, 4 sulphur cluster binding, lipoic acid \\
\hline F25P12.2 & Hydroxyproline-rich glycoprotein family protein \\
\hline MXE10.7 & $\mathrm{N}$-acetyltransferase activity metabolic process \\
\hline T4P13.22 & $\begin{array}{l}\text { Encodes an SNF1-related protein kinase that physically interacts with the SCF subunit SKP1/ASK1 and the } 20 \text { S proteosome subunit } \\
\text { PAD1. It can also interact with PRL1 DWD-containing protein. Based on in vitro degradation assays and cul4CS and prl1 mutants, there is } \\
\text { evidence that AKIN10 is degraded in a proteasome-dependent manner and that this depends on a CUL4-PRL1 E3 ligase }\end{array}$ \\
\hline
\end{tabular}

Interestingly, regulatory elements on the promoter region of $H K T$ in wild genotype have more frequency that cultivated genotype (mean of 2.28 regulatory element per unit compared to 1.92 regulatory element per unit). More importantly, regulatory elements are more variable

Table 7 Results of the pathway discovery analysis indicating proteins present and activated in the AtHKT1;1 pathway in the ATTED database

\begin{tabular}{ll}
\hline Gene or ID & Function \\
\hline TPS14 & Terpene synthase 14 \\
CYP71B31 & Mono oxygenase oxidoredoctasee \\
F15E12.22 & Acyl-activating enzyme 11 \\
F5D21.19 & Lipase class 3 family protein \\
MLP168 & Defence response \\
MYB21 MYB3 & Lipase class 3 family protein \\
MYB24 & DNA binding-jasmonic \& giberellic acid response \\
At3g14380 & Integral membrane family protein \\
At5g49130 & MATE efflux family protein \\
AtUGT85A3 & Glucuronosyl transferase \\
CYP76C3 & Electron carrier activity \\
At1g29490 & Auxin-responsive family protein \\
At3g55870 & Anthranilate synthase, alpha subunit, putative \\
AtMYB57 & DNA binding-jasmonic \& giberellic acid response \\
At5g05280 & Zinc finger (C3HC4-type RING finger) family protein \\
At2g17845 & Short-chain dehydrogenase/reductase (SDR) family protein \\
At3g23460 & Cyclopropane fatty acid synthase-related \\
At3g44250 & Electron carrier/ heme binding / monooxygenase/ \\
At1g13070 & Putative cytochrome P450 \\
\hline & \\
\hline
\end{tabular}

in wild wheat compared to cultivate wheat (variance of 19.54 verses 11.55 ).

It has been discussed that compared to cultivated genotypes, wild genotypes has better understanding from environment in com environment which helps them to manage energy more efficiently [30]. As example, before stress, wild genotypes keep the expression of mitochondrial ATP synthase lower than cultivated genotypes and save a considerable amount of energy. In contrast, early in stress, a sharp increase in activation and expression of ATP synthase happens in wild genotypes to cope with stress where this expression is much higher than cultivate genotypes [30]. The mentioned highly efficient energy consumption system needs a fast and reliable understanding of environmental conditions and signalling pathways.

Regulatory elements on the promoter region are the central inter-mediators which understand the environmental conditions from one site and activate transcription of genes on the other site. In fact, regulatory

Table 8 Descriptive statistics of regulatory elements on the promoter region of TmHKT1;5 (wild wheat) compared to TaHKT1;5 (bread wheat)

\begin{tabular}{lccccccc}
\hline & \multicolumn{2}{c}{$\begin{array}{c}\text { Central } \\
\text { tendency }\end{array}$} & & \multicolumn{5}{c}{ Variation tendency } \\
\cline { 2 - 3 } Gene & Mean & Median & & StDev & Variance & CV $^{\mathbf{2}}$ & Range \\
\hline $\begin{array}{l}\text { TmHKT1;5 } \\
\text { (wild wheat) }\end{array}$ & 2.28 & 1.00 & & 4.42 & 19.54 & 193.42 & 22.00 \\
$\begin{array}{l}\text { TaHKT1;5 } \\
\text { (bread wheat) }\end{array}$ & 1.92 & 1.00 & & 3.39 & 11.55 & 176.22 & 18.00 \\
\hline
\end{tabular}

${ }^{1}$ Standard deviation.

${ }^{2}$ Cofficient of variance. 
elements are the developed model of 2-component systems in bacteria.

It can be concluded that the higher frequency and higher variation of the regulatory elements on the promoter region of wild genotype provides the opportunity to respond to more elicitors and achieve the better understanding of environmental conditions. In other words, high efficiency in arrangement of regulatory elements on the promoter region guaranties higher reaction and better energy management. Additionally, higher frequency of specific regulatory elements such as CAAT-box (Table 1) contributes in rapid activation of transcription by the promoter of wild genotype.

Regarding the facts that up to now, breeding programs have defined solely based on the coding genes and the key roles of non-coding regions such as promoters are neglected [13], it is highly possible that the promoter based resistance mechanisms have not been transferred to cultivated genotypes during breeding programs. Recently, we presented a novel gene discover approach irrespective of gene sequence/blast based on promoter structure. In this approach, organization of regulatory elements was used as a module for mining of the whole genome and discovery of genes with similar promoter architecture [46].

\section{Conclusion}

In this study, analysis of $H K T$ transporters was carried by a range of in silico methods. It is likely that specific promoter elements are active in different tissues due to the observed differences in $H K T \mathrm{~s}$ function in various tissues. Identification of regulatory elements helps to illustrate various functions of $H K T$ genes in plants. In silico synteny and pathway discovery were useful for identification and characterization of $H K T$ isoforms and unravelling the molecular networks which they participate.

Abbreviation

HKT: High affinity potassium transporters.

\section{Competing interests}

The authors declare that they have no competing interests.

\section{Authors' contributions}

MZB, EE and AN designed, and conducted the analyses. MZB, EE and AN wrote the manuscript. All authors read and approved the final manuscript.

\section{Author details}

${ }^{1}$ Biotechnology Institute, Shiraz University, Shiraz, Iran. ${ }^{2}$ Department of Crop Production \& Plant Breeding, College of Agriculture, Shiraz University, Shiraz, Iran. ${ }^{3}$ School of Molecular \& Biomedical Science, The University of Adelaide, Adelaide, Australia.

Received: 29 January 2014 Accepted: 10 September 2014 Published: 15 September 2014

\section{References}

1. Amtmann A, Sanders D: Mechanisms of $\mathrm{Na}^{+}$uptake by plant cells. Adv Bot Res 1999, 29:75-112
2. James RA, Davenport RJ, Munns R: Physiological characterization of two genes for $\mathrm{Na}+$ exclusion in durum wheat, $\mathrm{Nax} 1$ and Nax2. Plant Physiol 2006, 142(4):1537-1547.

3. Corratgé-Faillie C, Jabnoune M, Zimmermann S, Véry AA, Fizames $C$, Sentenac H: Potassium and sodium transport in non-animal cells: The Trk/Ktr/HKT transporter family.Cell. Mol. Life Sci 2010, 67:2511-2532.

4. Durell SR, Guy HR: Structural models of the KtrB, TrkH, and Trk1,2 symporters based on the structure of the KcsA K + channel. Biophys 1999, 77:789-807.

5. Marino-Ramirez L, Tharakaraman K, Bodenreider O, Spouge J, Landsman D: Identification of cis-regulatory elements in gene co-expression networks using A-GLAM. Methods Mol Biol 2009, 541:1-22.

6. Mcclean PE, Mamidi S, McConnel M, Chikara S, Lee R: Synteny mapping between common bean and soybean reveals extensive blocks of shared loci. BMC Genomics 2010, 11(184):1474-2164.

7. Alvo M, Liu Z, Williams A, Yauk C: Testing for mean and correlation changes in microarray experiments: an application for pathway analysis. BMC Bioinformatics 2010, 11:60.

8. Hauser F, Horie T: A conserved primary salt tolerance mechanism mediated by HKT transporters: a mechanism for sodium exclusion and maintenance of high $\mathrm{K}(+) / \mathrm{Na}(+)$ ratio in leaves during salinity stress. Plant Cell Environ 2010, 33(4):552-565.

9. Byrt CS: Genes for sodium exclusion in wheat, PhD thesis. Australia: The University of Adelaide; 2008:1-155.

10. Kotelnikova E, Ivanikova N, Kalinin A, Yuryev A, Daraselia N: Atlas of Signaling for Interpretation of Microarray Experiments. PLOS ONE 2010, 5(2):e9256. doi:10.1371/journal.pone.0009256.

11. Muffato M, Louis A, Poisnel CE, Crollius HR: A database and a browser to study gene synteny in modern and ancestral genomes. Genomicus 2012, 8:1119-1121.

12. Obayashi T, Kinoshita K, Nakai K, Shibaoka M, Hayashi S, Saeki M, Shibata D, Saito K, Ohta H, ATTED-II: A database of co-expressed genes and cis elements for dentifying co-regulated gene groups in Arabidopsis. Nucleic Acids Res 2007, 35:D863-D869.

13. Deihimi T, Niazi A, Ebrahimi M, Kajbaf K, Fanaee S, Bakhtiarizadeh MZ, Ebrahimie E: Finding the undiscovered roles of genes: an approach using mutual ranking of coexpressed genes and promoter architecture-case study: dual roles of thaumatin like proteins in biotic and abiotic stresses. Springer Plus 2012, 1:30.

14. James RA, Blake C, Byrt CS, Munns R: Major genes for $\mathrm{Na}+$ exclusion, Nax1 and Nax2 (wheat HKT1;4 and HKT1;5), decrease $\mathrm{Na}+$ accumulation in bread wheat leaves under saline and waterlogged conditions. Exp Bot 2011, 62(8):2939-47.

15. Zamani Babgohari M, Niazi A, Aboli-moghaddam AA, Deihimi D, Ebrahimie $\mathrm{E}$ : Genome-wide analysis of key salinity-tolerance transporter $(H K T 1 ; 5)$ in wheat and wild wheat relatives (A and D genomes). In Vitro Cell Dev Biol-Plant 2012, 49:97-106.

16. Molina C, Grotewold E: Genome wide analysis of Arabidopsis core promoters. BMC Genomics 2005, 6:25.

17. Ashok Reddy D, Prasad BVLS, Mitra Chanchal K: Comparative analysis of core promoter region: Information content from mono and dinucleotide substitution matrices. Comput Biol Chem 2005, 30:58-62.

18. Joshi CP: An inspection of the domain between putative TATA box and translation start site in 79 plant genes. Nucleic Acids Res 1987, 15:16.

19. Grace ML, Chandrasekharan MB, Hall TC, Crow AJ: Sequence and Spacing of TATA Box Elements Are Critical for Accurate Initiation from the Phaseolin Promoter. Biol Chem 2004, 279(9):8102-8110.

20. Butler JE, Kadonaga JT: The RNA polymerase II core promoter: a key component in the regulation of gene expression. Genes Dev 2002, 16:2583-2592.

21. Sharma R, Katoch M, Govindappa N, Srivastava PS, Sastry K, Nabi Qazi G: Evaluation of the catalase promoter for expressing the alkaline xylanase gene (alx) in Aspergillus niger. FEMS Microbiol Lett 2011, 327:33-40.

22. Byrt CS, Platten JD, Spielmeyer W, James RA, Lagudah ES, Dennis ES, Tester M, Munns R: HKT1;5-like cation transporters linked to $\mathrm{Na}+$ exclusion loci in wheat, Nax2 and Kna1. Plant Physiol 2007, 143(4):1918-28.

23. Kusnetsov V, Landsberger M, Meurer J, Oelmüller R: The assembly of the CAAT-box binding complex at a photosynthesis gene promoter is regulated by light, cytokinin, and the stage of the Plastids. Biol Chem 1999, 274(50):36009-36014.

24. Menke FLH, Champion AW, Kijne J, Memelink J: A novel jasmonate- and elicitor-responsive element in the periwinkle secondary metabolite 
biosynthetic gene Str interacts with a jasmonate- and elicitorinducible AP2-domain transcription factor ORCA2. EMBO 1999, 18(16):4455-4463.

25. Lehmann J, Atzorn R, Brückner C, Reinbothe S, Leopold J, Wasternack C, Parthier B: Accumulation of jasmonate, abscisic acid, specific transcripts and proteins in osmotically stressed barley leaf segments. Planta 1995, 197:156-162.

26. del Amor FM, Cuadra-Crespo P: Alleviation of salinity stress in broccoli using foliar urea or methyl-jasmonate: analysis of growth, gas exchange, and isotope composition. Plant Growth Regul 2010, 63:55-62.

27. Platten JD, Cotsaftis O, Berthomieu P, Bohnert H, Davenport RJ, Fairbairn DJ, Horie T, Leigh RA, Lin HX, Luan S, Maser P, Pantoja O, Rodriguez-Navarro A, Schachtman DP, Schroeder JI, Sentenac H, Uozumi N, Very A, Zhu JK, Dennis ES, Tester M: Nomenclature for HKT transporters, key determinants of plant salinity tolerance. Trends Plant Sci 2006, 11(8):372-374.

28. Mäser $\mathrm{P}, \mathrm{Gierth} M$, Schroeder II: Molecular mechanisms of potassium and sodium uptake in plants. Plant Soil 2002, 247:43-54.

29. Mundy J, Chua NH: Abscisic acid and water-stress induce the expression of a novel rice gene. EMBO 1988, 7(8):2279-86.

30. Moghadam AA, Ebrahimie E, Taghavi SM, Niazi A, Zamani Babgohari M, Deihimi T, Djavaheri M, Ramezani A: How the nucleus and mitochondria communicate in energy production during stress: Nuclear MtATP6, an early-stress responsive gene, regulates the mitochondrial F1F0-ATP synthase complex. Mol Biotechnol 2013, 54:756-769.

31. Plett D, Safwat G, Gilliham M, Moller IS, Roy S, Shirley N, Jacobs A, Johnson A, Tester M: Improved salinity tolerance of rice through cell type-specific expression of AtHKT1;1. PLOS One 2010, 5:e12571.

32. Ren ZH, Gao JP, Li LG, Cai XL, Huang W, Chao DY, Zhu MZ, Wang ZY, Luan S, Lin HX: A rice quantitative trait locus for salt tolerance encodes a sodium transporter. Nat Genet 2005, 37:1141-1146.

33. Kader MA, Seidel T, Golldack D, Lindberg S: Expressions of OsHKT1, OsHKT2, and OsVHA are differentially regulated under $\mathrm{NaCl}$ stress in salt-sensitive and salt-tolerant rice (Oryza sativa L.) cultivars. Exp Bot 2006, 57:4257-4268

34. Waters S, Gilliham M, Hrmova M: Plant high-affinity potassium (HKT) transporters involved in salinity tolerance: structural insights to probe differences in lon Selectivity. Mol Sci 2013, 14:7660-7680.

35. Meijer AH, Scarpella E, van Dijk EL, Qin L, Taal AJ, Rueb S, Harrington SE, McCouch SR, Schilperoort RA, Hoge JH: Transcriptional repression by Oshox1, a novel homeodomain leucine zipper protein from rice. Plant 1997, 11(2):263-76.

36. G'omez-Cadenas A, TadeoFR TM, Primo-Millo E: Leaf abscission induced by ethylene in water-stressed intact seedlings of cleopatra mandarin requires previous abscisic acid accumulation in roots. Plant Physiol 1996, 112:401-408.

37. Arbona V, Argamasilla R, G'omez-Cadenas A: Common and divergent physiological, hormonal and metabolic responses of Arabidopsis thaliana and Thellungiella halophila to water and salt stress. Plant Physiol 2010, 167:1342-1350.

38. Lam E, Chua NH: Tetramer of a 21-base pair synthetic element confers seed expression and transcriptional enhancement in response to water stress and abscisic acid. Biol Chem 1991, 266(26):17131-5.

39. Mundy J, Yamaguchi-Shinozaki K, Chua NH: Nuclear proteins bind conserved elements in the abscisic acid-responsive promoter of a rice rab gene. Proc Natl Acad Sci 1990, 87(4):1406-10.

40. Zhu JK: Regulation of ion homeostasis under salt stress. Curr Opin Plant Biol 2003, 6(5):441-5.

41. Almeida P, Katschnig DH, de Boer D: HKT Transporters-State of the Art. Mol Sci 2013, 14:20359-20385.

42. Ramezani A, Niazi A, Abolimoghadam AA, Zamani Babgohari M, Deihimi T, Ebrahimi M, Akhtardanesh H, Ebrahimie E: Quantitative expression analysis of TaSOS1 and TaSOS4 genes in cultivated and wild wheat plants under salt stress. Mol Biotechnol 2012, 53:189-197.

43. Moon A, Prinsen E, Bauw G, Van Montagu M: Antagonistic effects of abscisic acid and jasmonates on salt stress-Inducible transcripts in rice roots. Plant Cell 1997, 9:2243-2259.

44. Ebrahimie M, Esmaeili F, Cheraghi S, Houshmand F, Shabani L, Ebrahimie E: Efficient and Simple Production of Insulin-Producing Cells from Embryonal Carcinoma Stem Cells Using Mouse Neonate Pancreas Extract, As a Natural Inducer. PLOS ONE 2014, 9:e90885.
45. Obayashi T, Kinoshita K: Rank of correlation coefficient as a comparable measure for biological significance of gene coexpression. DNA Res 2009, 16:249-60.

46. Hosseinpour B, Bakhtiarizadeh MR, Khosravi P, Ebrahimie E: Predicting distinct organization of transcription factor binding sites on the promoter regions: a new genome-based approach to expand human embryonic stem cell regulatory network. Gene 2013, 531:212-219.

doi:10.1186/2046-9063-10-9

Cite this article as: Zamani Babgohari et al:: In silico analysis of high affinity potassium transporter (HKT) isoforms in different plants. Aquatic Biosystems 2014 10:9.

\section{Submit your next manuscript to BioMed Central and take full advantage of:}

- Convenient online submission

- Thorough peer review

- No space constraints or color figure charges

- Immediate publication on acceptance

- Inclusion in PubMed, CAS, Scopus and Google Scholar

- Research which is freely available for redistribution 